\title{
AutódRomos AMBIENTALMENTE SUSTENTABLES: ELEMENTOS PARA EL CONTROL ACTIVO Y PASIVO DEL RUIDO
}

\author{
ENVIRONMENTALLY SUSTAINABLE RACETRACKS: PASSIVE AND ACTIVE NOISE \\ CONTROL ELEMENTS \\ Luis Fernando Molina-Prieto* \\ Giovanni González Torres** \\ Hugo Andrés Perilla Jácome ${ }^{* * * *}$
}

Recibido: 11 de enero de 2017

Aceptado: 25 de abril de 2017

\section{Resumen}

El artículo aborda un problema contemporáneo que afecta a miles de ciudades del mundo: el ruido, específicamente, el ruido que generan las competencias de deportes a motor en los autódromos. Se esboza la situación de algunas pistas de diversos países que se han visto limitadas e, incluso, clausuradas por no cumplir con las normas de ruido exigidas por las autoridades ambientales. Tomando como marco teórico el control del ruido, se indaga en dos vertientes bien diferenciadas: el control pasivo del ruido y el control activo del ruido. Además y considerando que la normativa concerniente al ruido dista de ser homogénea a nivel internacional, se realiza una pesquisa inicial en ese sentido y se presentan sus resultados; con base en ellos, se busca elevar una solicitud formal a la autoridad ambiental colombiana para que modifique el umbral de ruido permitido en torno a los autódromos.

Palabras clave: autódromos, control activo del ruido, control pasivo del ruido.

\section{Abstract}

The article addresses a contemporary problem affecting thousands of cities in the world: noise, specifically, the intense noise generated by the motor sports competitions in the racetracks. It outlines the situation of some tracks in various countries, which have been limited or even closed, for not complying with noise standards required by environmental authorities. Taking as a theoretical

\footnotetext{
* Arquitecto. Docente investigador, Grupo de investigación Territorio y Habitabilidad, Fundación Universidad de América. Imolinaprieto@gmail.com

** Director de investigaciones CESVI Colombia S. A, Centro de Experimentación y Seguridad Vial de Colombia. ggonzalez@cesvicolombia.com

*** Gerente general Autódromo XRP Motorpark Cajicá. ha.perilla@xrpcolombia.com
} 
framework the control of noise, two well-differentiated aspects are investigated: passive noise control and active noise control. In addition, and considering that the rules concerning noise is far from homogeneous at the international level, an initial research is carried out in this sense, and their results are presented. Based on these, it's seeks to raise a formal request to the Colombian environmental authority to modify the noise threshold allowed around the racetracks.

Keywords: racetracks, active noise control, passive noise control.

\section{INTRODUCCIÓN}

El nacimiento de los deportes a motor tuvo lugar en Francia en 1894, cuando se realizó una competencia entre París y Rouen (patrocinada por la revista Le Petit Journal), la cual, más que una carrera, fue una puesta a punto de los vehículos que participaron, en la que se valoró la confiabilidad y el rendimiento de los autos. Un año después se llevó a cabo otra competencia entre París y Bordeaux, esta sí enfocada en la velocidad y con una línea de meta que permitió establecer un ganador. Esas primeras competencias realizadas en vías y caminos públicos generaron una serie de accidentes que causaron heridas y muertes a peatones, caballos y competidores, por lo que se percibieron como actividades de gran peligrosidad. No obstante, continuaron realizándose en distintos lugares de Europa. En 1903, durante la carrera París-Madrid, al menos seis personas murieron y decenas resultaron heridas. Por este motivo surgió la idea de realizar las competencias a motor en circuitos cerrados, utilizando para ello vías existentes que se clausuraban durante la carrera. Esta nueva condición para ese tipo de competencias aportaba mayor seguridad a pilotos y espectadores y no ponía en riesgo al público en general. Tres años después, esos primigenios desarrollos concluyeron en el Grand Prix de Francia; carrera para la que se cerraron algunas calles de la ciudad de Le Mans (Seneca, 2003). Desde entonces, los deportes de motor han alcanzado niveles de desarrollo verdaderamente sofisticados, entre los que se encuentra la construcción de escenarios especialmente diseñados para tales actividades: los autódromos. Estos espacios se caracterizan por ser: i) escenarios idóneos para las competencias (por su seguridad); ii) polos de atracción turística que generan empleo y traen grandes dividendos económicos, y iii) ambientes de experimentación y desarrollo tecnológico al servicio de la industria automotriz a nivel global.

Entre los factores por los que se cuestiona a los autódromos, todos ellos relacionados con su funcionamiento, como la concentración de residuos sólidos contaminantes (por ejemplo, la gran cantidad de llantas usadas que se generan con las competencias), los elevados niveles de contaminación del aire que producen los motores de combustión interna, o los riesgos asociados a la concentración de combustibles y carburantes en esos escenarios deportivos, son los altos niveles de ruido los que crean los más graves problemas. Esto se evidencia en las numerosas demandas que se interponen en contra de los autódromos por ese motivo. En Colombia y otros países, estas acciones legales han logrado: i) establecer restricciones para el horario de las competencias; ii) cerrar temporalmente los autódromos mientras se realizan labores que minimicen la dispersión del ruido en el entorno; iii) establecer un número máximo de competencias por año, mes o semana, y iv) cerrar de manera definitiva estos escenarios deportivos (Carrado, 2015; Cheng y Jarvis, 2010; Kardous y Morata, 2010). A continuación se describen algunos casos destacables.

Argentina. E1 Autódromo Ciudad de Rosario, pese a contar con una larga trayectoria en la ciudad y ser un lugar que congrega a distintos sectores de la sociedad, y que además, dinamiza la economía local y genera numerosos puestos de trabajo, debió acatar la Ordenanza n. 8934 de 2012 de la Municipalidad de Rosario, que le impuso: realizar obras para mitigar la dispersión del ruido; efectuar como máximo ocho carreras anuales, y desarrollar las pruebas de puesta a punto 
únicamente dos días hábiles por semana, con silenciadores y en un horario específico, entre las 8:00 y 13:00 horas y las 15:00 y 18:00 horas (Consejo Municipal de Rosario, Ordenanza 8934).

Canadá. El Autódromo de Saint-Eustache (Quebec) debió construir en 2011 un muro en toda la extensión de su perímetro para evitar la dispersión del ruido generado durante las competencias. Pero eso no fue suficiente: en agosto de 2013, la administración local exigió que este escenario se sometiera a la supervisión continua de un comité de vigilancia conformado por un grupo de vecinos de la pista y representantes del autódromo (Côté, 2013).

Chile. El Autódromo Internacional de Codegua, tras una inversión de 25 millones de dólares, debió cerrarse un mes y medio después de su inauguración por no cumplir con la normativa ambiental en el tema del ruido, que en ese país no debe superar los 51 decibeles. Aunque la Corte Suprema de Chile rechazó el recurso de protección presentado por un grupo de vecinos del autódromo, se declaró incompetente en asuntos medioambientales, de manera que transfirió la responsabilidad y la decisión a la Superintendencia del Medio Ambiente, que decidió clausurar el escenario deportivo hasta que se construyeran las barreras necesarias para que el ruido se mantuviera dentro de los umbrales permitidos (Gana, 2014). Las obras se ejecutaron; pero en enero de 2016 el autódromo fue nuevamente clausurado por no cumplir con la normativa en cuanto a ruido (Paredes, 2016).

Colombia. Las competencias deportivas en los autódromos de Colombia han sido limitadas reiteradamente por tutelas, demandas y otras acciones judiciales. La Sentencia T-226 de 1995 prohibió realizar eventos nocturnos en el Autódromo de Tocancipá (excepto en el caso de carreras internacionales, "cuya realización comprenda un período nocturno a lo largo del año" y que no pasen de las 10:00 p. m.) (Corte Constitucional, Sentencia T-226).

E1 Autódromo XRP Motorpark de Cajicá está localizado al norte de este municipio, en una zona que el Plan de Ordenamiento Territorial (POT) estableció como área recreativa e institucional. El autódromo de Cajicá se ajusta a la normativa territorial vigente y se encuentra en funcionamiento. No obstante, debido al ruido, una acción popular pretendió obstaculizar su funcionamiento; pero el Consejo de Estado profirió el 7 de julio de 2015 un fallo de última instancia a favor del autódromo. ${ }^{1}$ Así, según la Corte, los problemas derivados del ruido generado por los deportes a motor se consideran un caso juzgado y, por tanto, plenamente resuelto, fallado y cerrado, pues la legislación colombiana impide juzgar dos veces un mismo caso. Si bien el Autódromo de Cajicá no tiene inconvenientes en los campos normativo y jurídico, actualmente está obligado a reducir el umbral de ruido a un metro de distancia de su perímetro, a un máximo de 55 decibeles.

Es importante subrayar que los autódromos inciden de manera positiva en una serie de dimensiones: i) fortalecen los deportes a motor y los desarrollos tecnológicos en el ámbito automotor; ii) incrementan las dinámicas económicas en su área de influencia; iii) generan tejido y cohesión social; iv) establecen importantes polos de atracción turística; v) generan dinámicas económicas y atraen divisas, contribuyendo significativamente a la sustentabilidad económica de los municipios, las ciudades y los países en donde se asientan, y vi) son los únicos lugares idóneos para ejercitar los deportes a motor en condiciones seguras, tanto para los pilotos, como para el público en general (Añó, Bastías y Pablos, 2010; Henderson, Foo, Lim y Yip, 2010). Por el contrario, la ausencia de autódromos genera una serie de efectos negativos e, incluso, desastrosos: i) los aficionados a los deportes de motor se exponen a riesgos de consecuencias funestas; ii) se multiplican los riesgos de accidentalidad en las ciudades y carreteras; iii) se incrementa el número de pérdidas sociales y vidas humanas; iv) se elevan las pérdidas económicas por accidentes de tráfico, y v) las ciudades y

${ }^{1}$ Expediente 25000-23-24-000-2011-00546-01 
carreteras reducen notoriamente sus márgenes de seguridad y sostenibilidad. En pocas palabras: los autódromos minimizan los riesgos de accidentalidad, generan riqueza, turismo, tejido social e innovaciones tecnológicas; pero producen ruido y concentración de residuos. En consecuencia, la investigación busca moderar la gran limitante de los autódromos y las pistas: el ruido.

En este contexto y teniendo en cuenta que algunos autores señalan la existencia de considerables dudas sobre si en la actualidad se gestionan en una forma ambientalmente sostenible las actividades en los autódromos (Dingle, 2009; Kardous y Morata, 2010; Tranter, 2003), el artículo busca aportar, en lo concerniente al ruido, el componente de sustentabilidad que requieren con urgencia estos escenarios deportivos, como es el diseño y desarrollo de sistemas, dispositivos y artefactos que permitan controlar la dispersión del ruido en los entornos mediato e inmediatos (barreras naturales, barreras artificiales y sistemas de control activo del ruido). Adicionalmente, considerando que la normativa ambiental en el tema de la contaminación auditiva resulta en ocasiones incongruente o inconsecuente con las actividades que se desarrollan en los autódromos, puesto que, de por sí, efectivamente producen ruido, se valoran las normativas colombianas e internacionales en cuanto a los decibeles permitidos en torno los autódromos, para que se motive, impulse y gestione la modificación de los umbrales mínimos de ruido permitidos por parte de la autoridad ambiental colombiana.

\section{Marco teórico}

Este artículo se enmarca en el concepto del control del ruido; ámbito temático de carácter teóricopráctico que involucra los campos de la salud física y mental de los seres humanos expuestos al ruido, así como las normativas internacionales que establecen los niveles permitidos de ruido ambiental. El control del ruido fue estudiado en el siglo XX en dos tipos de ambientes: los industriales (Bies y Hansen, 2009; Cai et ál., 2013) y los urbanos (Tsai, Lin y Chen 2009; Yang y Kang, 2005; Yepes, Gómez, Sánchez y Jaramillo, 2009), e involucra: el estudio del ruido y su propagación; el análisis de las ondas sonoras y su velocidad, densidad espectral y medición; además de variables relacionadas con la percepción humana de los sonidos, como las sensaciones psicoacústicas, la sensación de altura y la sensación de sonoridad, entre muchas otras (Miyara, 1999).

El objeto de estudio del artículo, la mitigación del ruido producido por los motores de combustión interna, se focaliza e intensifica en los autódromos; pero es común en las grandes ciudades, especialmente en las zonas de alto tráfico (Zannin y de Sant'Ana, 2011; Hunashal y Patil, 2012), así como en los aeropuertos (Vogiatzis, 2012), y es considerado uno de los mayores problemas ambientales que enfrentan las ciudades contemporáneas (Germán-González y Santillán, 2006). Por tanto, los resultados tanto teóricos como tecnológicos y de diseño que genere esta investigación serán de gran utilidad y aplicabilidad. En un primer momento, en el área de estudio (autódromo de Cajicá), donde serán monitoreados y evaluados sus alcances y limitaciones; en un segundo momento, en otros autódromos (del país, el continente y el mundo); y en un tercer momento, en ámbitos en los que el ruido generado por los automotores supere los estándares autorizados.

\section{Área de estudio}

El área de estudio es el Autódromo XRP Motorpark de Cajicá (figura 1). Allí se realizarán mediciones de intensidad de ruido durante las competencias de tres tipos de vehículos: karts, motocicletas y automóviles. Asimismo, en el área de estudio se instalarán barreras naturales y artificiales, y se llevarán a cabo los experimentos de control activo del ruido. 


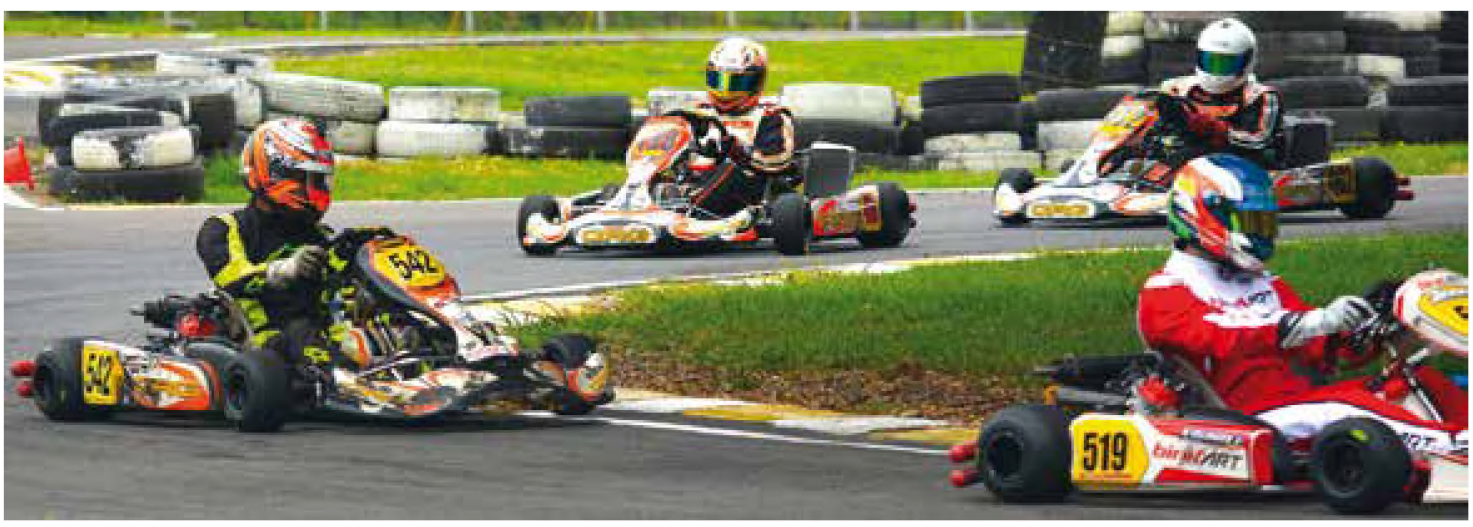

Figura 1. Área de estudio: autódromo XRP Motorpark de Cajicá.

Foto de Luis Fernando Molina-Prieto (agosto de 2016).

\section{Metodología}

Tras una primera revisión de la literatura no se encontraron estudios similares al que propone el artículo. En el campo de las ciencias de la salud se han publicado algunos artículos enfocados a los efectos que genera el ruido en los pilotos profesionales y en el público que asiste a las competencias, pero no hay estudios centrados en el control activo y pasivo del ruido para evitar su propagación ambiental. Teniendo en cuenta lo anterior, se plantea la siguiente metodología.

a. Revisión bibliográfica. Se revisarán dos tipos de documentos: i) los que reporten barreras naturales y artificiales y cualquier otro tipo de artefactos que mitiguen la dispersión del ruido en entornos urbanos e industriales, y ii) los que, a partir del concepto del control activo del ruido, presenten resultados para neutralizar o anular ondas sonoras mediante contrasonidos o contrafrecuencias.

b. Trabajos de campo. Estos se realizarán en el área de estudio con el fin de alcanzar tres objetivos: i) elaborar una línea base ambiental en la matriz aire-ruido durante los eventos deportivos; ii) establecer las zonas más propicias para la plantación de barreras naturales y la instalación de barreras artificiales contra el ruido, y iii) caracterizar las diversas clases de vehículos que compiten en el autódromo en lo concerniente a sus niveles de producción de ruido.

c. Diseño de barreas naturales. A partir del estudio de los árboles de la Sabana de Bogotá y teniendo en cuenta características propias de cada especie, como altura, densidad del follaje, índice de crecimiento, longevidad, sistema radicular y otras de carácter netamente vegetal, se creará una matriz para la selección de las especies más adecuadas para plantar en el área de estudio, con el fin de que sus troncos, ramas y follaje absorban y disgreguen las ondas sonoras y reduzcan la dispersión del ruido causado durante las competencias en el entorno circundante del escenario deportivo.

d. Diseño de barreas artificiales. A partir del análisis de las barreras artificiales para la reducción del ruido que actualmente se están instalando en corredores férreos y viales de países de Europa y Norteamérica, se harán propuestas de diseño de barreras artificiales contra el ruido, diseñadas y concebidas con materiales e insumos de fácil consecución en Colombia. Además, se establecerán los lugares específicos para su instalación en el área de estudio y se calcularán los sistemas de soporte requeridos para que su emplazamiento no genere ningún tipo de riesgo, ni al público del autódromo ni a los habitantes del sector.

e. Control activo del ruido. Se estudiarán documentos que den cuenta de los avances en lo concerniente al control activo del ruido y su aplicación, y se analizarán dispositivos diseñados 
especialmente para ese fin. Estos dispositivos, por lo general, están compuestos por un sensor (que valora el ruido ambiente), un procesador (que genera un contrasonido u onda sonora de fase inversa) y un emisor (que amplifica y dispersa en el aire el contrasonido, gracias a lo cual las ondas se interfieren y terminan por suprimirse mutuamente).

f. Valoración in situ. Se realizarán pruebas en el área de estudio con prototipos de los dispositivos para el control activo y pasivo del ruido.

g. Solicitud de cambio de normativa. A partir de los resultados de la investigación y si es pertinente, se elevará una petición a las autoridades competentes para que revisen la normativa que exige a los autódromos colombianos reducir sus emisiones de ruido a un máximo de 55 decibeles, a un metro de su perímetro. Asimismo, se solicitará la ampliación de este umbral por ser inconsistente con las políticas nacionales de seguridad automotriz, que exigen que los deportes a motor se realicen en pistas adecuadas, es decir, en autódromos; aunque tales políticas de seguridad no se ponen en práctica, porque las competencias generan ruido y la autoridad ambiental no las permite.

\section{Resultados}

\section{Control pasivo del ruido}

\section{Barreras naturales contra el ruido}

La contaminación acústica urbana genera diversas perturbaciones a los ciudadanos. Los viaductos crean cañones artificiales que incrementan el ruido de los automotores, porque las fachadas de los edificios son por lo general rígidas y generan altos niveles de reverberación (Thomas, Dragonetti, van Renterghem y Botteldooren, 2010). Las investigaciones de Fang y Ling (2003), así como las de Van Renterghem, Botteldooren y Verheyen (2012), entre otras, han evidenciado que las arborizaciones minimizan de manera significativa el ruido de los automotores, lo que se explica porque las superficies blandas de las hojas y la aleatoria disposición de las ramas y el follaje dispersan las ondas sonoras (en tanto que el suelo las absorbe), de modo que reducen la reverberación. Además y con base en sus trabajos de campo, estos investigadores han evidenciado que las franjas de árboles plantados paralelamente a las vías amortiguan significativamente el ruido. Por su parte, van Renterghem et ál. (2015) estudiaron las barreras naturales contra el ruido, entre ellas los cinturones de árboles y los taludes, mostrando que su eficiencia es especialmente significativa en distancias próximas a la fuente del ruido, pues conforman una zona de sombra acústica que se prolonga en relación directa con la altura, la porosidad y otras características de la barrera.

De otro lado, Maleki y Hosseini (2011) realizaron pruebas de control de ruido a partir de barreras de árboles en Teherán, Irán. Estos autores utilizaron para sus ensayos especies de árboles existentes en esa ciudad, que variaban notablemente en cuanto a su diversidad y tamaño, y valoraron como la barrera más eficiente contra el ruido la del monocultivo de la especie Robinia pseudoacasia, árbol muy utilizado en los espacios públicos de esa ciudad. Teniendo en cuenta estos resultados, para el área de estudio y experimentación se creará una matriz que permita seleccionar las especies de árboles más adecuados para absorber y disgregar las ondas sonoras, a partir de variables como altura, densidad del follaje, índice de crecimiento, longevidad, sistema radicular y otras de carácter netamente vegetal.

\section{Barreras artificiales contra el ruido}

En la actualidad existen empresas que ofrecen barreras acústicas fonoabsorbentes en diferentes materiales (incluso, transparentes), que permiten mitigar el ruido de vías y autopistas de alto tráfico. 
Serrano, Abad, Magro y García (2009) clasifican las barreras o pantallas acústicas en siete tipos de pantallas: hormigón, metálicas, transparentes, madera, mixtas, espuma de arcilla y ladrillos absorbentes. Por su parte, Pfretzschner, Simón, Rodríguez y Moreno (2010) valoraron el uso de granza de caucho en la construcción de pantallas acústicas absorbentes. Para el área de estudio se diseñaron pantallas constituidas con dos elementos: llantas usadas de karts respaldadas con tejas termoacústicas (ver figuras 2 y 3 ). A la fecha se han construido noventa metros lineales con este tipo de barrera.

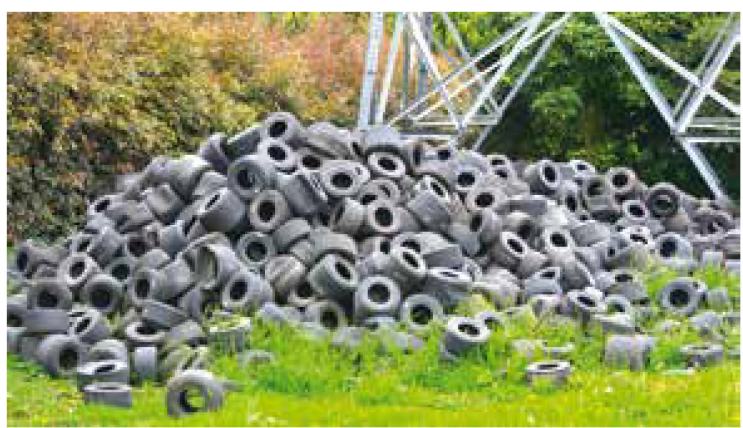

Figura 2. Acumulado de llantas de karts en el área de estudio.

Foto de Luis Fernando Molina-Prieto (agosto de 2016).

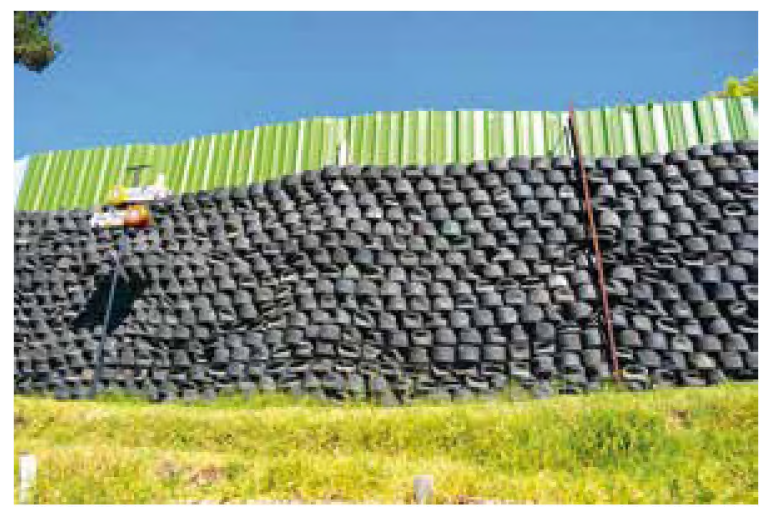

Figura 3. Construcción de barreras artificiales con llantas usadas de karts en el área de estudio.

Foto de Luis Fernando Molina-Prieto (octubre de 2016).

\section{Control activo del ruido}

La idea del control activo del ruido es la de generar y emitir una antifrecuencia que anule o destruya el ruido producido por un emisor (o el ruido que se encuentra en el ambiente). Este concepto fue propuesto originalmente en 1934 por el físico alemán Paul Lueg, quien lo patentó el 9 de junio de 1936 como Process of Silencing Sound Oscillations ${ }^{2}$. No obstante, fue solo hasta la década de 1980 cuando se presentaron aplicaciones prácticas, especialmente para conductos, como tubos de escape, sistemas de ventilación o aire acondicionado, y protectores auditivos. Una década después se dieron a conocer resultados muy interesantes para aplicar los principios del control activo del ruido en el interior de medios de transporte, como vehículos, trenes y aviones. Cabe subrayar que este

\footnotetext{
${ }^{2}$ Patente US2043416
} 
sistema es muy eficiente para contrarrestar frecuencias bajas, algo que el control pasivo del ruido (barreras naturales y artificiales) no logra. Por tanto, se utilizan comúnmente los sistemas activo y pasivo para una mejor reducción y control del ruido (Cobo, Bravo, Cuesta, Ranz y Siguero, 2000; Elliott y Nelson, 1993; Kuo y Morgan, 1999). El control activo del ruido actualmente se aplica en diversos dispositivos que detectan la amplitud y la frecuencia de ondas sonoras perturbadoras y emiten, en el mismo instante, un contrasonido u onda sonora de fase inversa que provoca que ambas ondas se interfieran y terminen por suprimirse mutuamente, como ocurre, por ejemplo, en algunas sillas de los aviones de pasajeros. De otro lado, la empresa israelita Silentium Ltd. desarrolló recientemente el "chip del silencio", como parte de la tecnología del control activo del ruido. Con estas tecnologías se busca desarrollar una burbuja silente entorno al área de estudio, o reducir el ruido al emitir contrafrecuencias en tiempo real, que eliminen el ruido generado por las competencias de motor en el escenario deportivo.

\section{Normativa vigente}

A continuación se presenta un resumen sucinto de las normativas concernientes al ruido en tres diferentes niveles: nacional, regional e internacional.

\section{Normas nacionales}

Resolución 8321 del 4 de agosto de 1983 expedida por el Ministerio de Salud. El artículo 17 establece cuatro zonas receptoras de ruido y cuatro niveles máximos de decibeles $(\mathrm{dB})$ permitidos:

- Zona I residencial: $65 \mathrm{~dB}$ diurnos y $45 \mathrm{~dB}$ nocturnos.

- Zona II comercial: $70 \mathrm{~dB}$ diurnos y $60 \mathrm{~dB}$ nocturnos.

- Zona III industrial: $75 \mathrm{~dB}$ diurnos y $75 \mathrm{~dB}$ nocturnos.

- Zona IV de tranquilidad: $45 \mathrm{~dB}$ diurnos y $45 \mathrm{~dB}$ nocturnos.

Los artículos 19, 20 y 21 del capítulo II estipulan protocolos detallados para la medición del ruido (Resolución 8321,1983 ).

Decreto 948 del 5 de junio de 1995 proferido por el Ministerio del Medio Ambiente. El artículo 15 establece cuatro sectores con restricciones en cuanto a ruido ambiental, pero no define el nivel de decibeles permitido para ninguno de ellos.

1. Sectores A. (Tranquilidad y silencio): áreas urbanas donde estén situados hospitales, guarderías, bibliotecas, sanatorios y hogares geriátricos.

2. Sectores B. (Tranquilidad y ruido moderado): zonas residenciales o exclusivamente destinadas para desarrollo habitacional, parques en zonas urbanas, escuelas, universidades y colegios.

3. Sectores C. (Ruido intermedio restringido): zonas con usos permitidos industriales y comerciales, oficinas, uso institucional y otros usos relacionados.

4. Sectores D. (Zona suburbana o rural de tranquilidad y ruido moderado): áreas rurales habitadas destinadas a la explotación agropecuaria, o zonas residenciales suburbanas y zonas de recreación y descanso (Decreto 948, 1995).

Resolución 627 de abril 7 de 2006 expedida por el Ministerio de Ambiente, Vivienda y Desarrollo Territorial. El artículo 9 define los estándares máximos permitidos para niveles de emisión de ruido en cuatro sectores (ver tabla 1). Además, el artículo aclara que: "Cuando la emisión de ruido en un sector o subsector, trascienda a sectores o subsectores vecinos o inmersos en él, los estándares máximos permisibles de emisión de ruido son aquellos que corresponden al sector o subsector más restrictivo" (Resolución 627, 2006, art. 9, par. 1). 
Tabla 1. Estándares máximos permisibles de niveles de emisión de ruido en Colombia expresados en decibeles $\mathrm{dB}$

\begin{tabular}{|c|c|c|c|}
\hline \multirow[t]{2}{*}{ Sector } & \multirow[t]{2}{*}{ Subsector } & \multicolumn{2}{|c|}{$\begin{array}{l}\text { Estándares máximos } \\
\text { permisibles de niveles de } \\
\text { emisión de ruido en dB }\end{array}$} \\
\hline & & Día & Noche \\
\hline $\begin{array}{l}\text { Sector A. Tranquilidad y } \\
\text { silencio }\end{array}$ & $\begin{array}{l}\text { Hospitales, bibliotecas, guarderías, sanatorios, hogares } \\
\text { geriátricos. }\end{array}$ & 55 & 50 \\
\hline \multirow{3}{*}{$\begin{array}{l}\text { Sector B. Tranquilidad y } \\
\text { ruido moderado }\end{array}$} & $\begin{array}{l}\text { Zonas residenciales o exclusivamente destinadas para } \\
\text { desarrollo habitacional, hotelería y hospedajes. }\end{array}$ & & \\
\hline & $\begin{array}{l}\text { Universidades, colegios, escuelas, centros de estudio e } \\
\text { investigación. }\end{array}$ & 65 & 55 \\
\hline & $\begin{array}{l}\text { Parques en zonas urbanas diferentes a los parques } \\
\text { mecánicos al aire libre. }\end{array}$ & & \\
\hline \multirow{4}{*}{$\begin{array}{l}\text { Sector C. Ruido intermedio } \\
\text { restringido }\end{array}$} & $\begin{array}{l}\text { Zonas con usos permitidos industriales, como } \\
\text { industrias en general, zonas portuarias, parques } \\
\text { industriales, zonas francas. }\end{array}$ & 75 & 75 \\
\hline & $\begin{array}{l}\text { Zonas con usos permitidos comerciales, como centros } \\
\text { comerciales, almacenes, locales o instalaciones de } \\
\text { tipo comercial, talleres de mecánica automotriz e } \\
\text { industrial, centros deportivos y recreativos, gimnasios, } \\
\text { restaurantes, bares, tabernas, discotecas, bingos, } \\
\text { casinos. }\end{array}$ & 70 & 60 \\
\hline & $\begin{array}{l}\text { Zonas con usos permitidos de oficinas. } \\
\text { Zonas con usos institucionales. }\end{array}$ & 65 & 55 \\
\hline & $\begin{array}{l}\text { Zonas con otros usos relacionados, como parques } \\
\text { mecánicos al aire libre, áreas destinadas a } \\
\text { espectáculos públicos al aire libre. }\end{array}$ & 80 & 75 \\
\hline $\begin{array}{l}\text { Sector D. Zona suburbana } \\
\text { o rural de tranquilidad y } \\
\text { ruido moderado }\end{array}$ & $\begin{array}{l}\text { Residencial suburbana. } \\
\text { Rural habitada destinada a explotación agropecuaria. } \\
\text { Zonas de Recreación y descanso, como parques } \\
\text { naturales y reservas naturales. }\end{array}$ & 55 & 50 \\
\hline
\end{tabular}

Fuente: Ministerio de Ambiente, Vivienda y Desarrollo Territorial (Resolución 627, 2006, art. 9).

E1 capítulo IV de la Resolución 627 de 2006 define los equipos de medida (art. 18), las calibraciones (art. 19), las condiciones meteorológicas exigidas (art. 20), los contenidos de los informes técnicos (art. 21) y la obligatoriedad de la realización de mapas de ruido por parte de las corporaciones autónomas regionales (arts. 22 y 24), así como los fines y contenidos de los mapas de ruido (art. 23) y los requisitos mínimos que se deben cumplir durante su elaboración (art. 24). Además, en el anexo 3 se establecen los procedimientos para la medición de emisiones de ruido (capítulo I), los procedimientos para la medición del ruido ambiental (capítulo II) y el procedimiento para la determinación del número de puntos y los tiempos de medición para ruido ambiental (capítulo III). Por último, cabe subrayar que en el anexo 5 se establece de manera rigurosa la representación de resultados en los mapas de ruido, lo que incluye los colores correspondientes a las diferentes zonas de ruido medidas en decibeles. 


\section{Normas regionales}

Resolución 6918 de 2010 expedida por la Secretaria Distrital de Ambiente de Bogotá. El artículo 7 establece los valores máximos permisibles de ruido en el interior de las edificaciones receptoras:

- Edificaciones de uso residencial: $55 \mathrm{~dB}$ diurnos y $45 \mathrm{~dB}$ nocturnos.

- Edificaciones de uso institucional (oficinas públicas y/o privadas): $55 \mathrm{~dB}$ diurnos y $45 \mathrm{~dB}$ nocturnos.

- Edificaciones de usos dotacionales contempladas en el POT: $55 \mathrm{~dB}$ diurnos y $45 \mathrm{~dB}$ nocturnos.

- Áreas comunes en edificaciones destinadas a actividades comerciales: $70 \mathrm{~dB}$ diurnos y $70 \mathrm{~dB}$ nocturnos (Resolución 6918, 2010, art. 7).

Convenio 543 de 2008 expedido por el Area Metropolitana de Valle de Aburrá. Esta normativa se acoge a los estándares permitidos de ruido establecidos a nivel nacional, o sea, a lo establecido por la Resolución 627 de 2006 (ver tabla 1) (Convenio 543, 2008).

\section{Normas internacionales}

Decreto Supremo n. 085-2003-PCM expedido por la Presidencia de la República del Perú. Estándares nacionales de calidad ambiental para ruido:

- Zona de protección especial: $50 \mathrm{~dB}$ diurnos y $40 \mathrm{~dB}$ nocturnos.

- Zona residencial: $60 \mathrm{~dB}$ diurnos y $50 \mathrm{~dB}$ nocturnos.

- Zona comercial: $70 \mathrm{~dB}$ diurnos y $60 \mathrm{~dB}$ nocturnos.

- Zona industrial: $80 \mathrm{~dB}$ diurnos y $70 \mathrm{~dB}$ nocturnos (Decreto Supremo n. ${ }^{\circ} 085,2003$ ).

Resolución n 0123 expedida por la Dirección Metropolitana de Medioambiente de Quito. Niveles máximos permitidos de ruido para fuentes fijas:

- Zona equipamientos y protección: $45 \mathrm{~dB}$ diurnos y $35 \mathrm{~dB}$ nocturnos.

- Zona residencial: $50 \mathrm{~dB}$ diurnos y $40 \mathrm{~dB}$ nocturnos.

- Zona residencial múltiple: $55 \mathrm{~dB}$ diurnos y $45 \mathrm{~dB}$ nocturnos.

- Zona industrial 1: $60 \mathrm{~dB}$ diurnos y $50 \mathrm{~dB}$ nocturnos.

- Zona industrial 2: $65 \mathrm{~dB}$ diurnos y $55 \mathrm{~dB}$ nocturnos.

- Zona industrial 3 y 4: $70 \mathrm{~dB}$ diurnos y $60 \mathrm{~dB}$ nocturnos (Resolución n. ${ }^{\circ} 0123,2004$ ).

Tabla 2. Límites máximos permisibles de ruido en Buenos Aires

\begin{tabular}{lcc}
\hline & \multicolumn{2}{c}{ Valores límite en dB } \\
\hline Área de sensibilidad acústica & Período diurno (15 h) & Período nocturno (9 h) \\
\hline Tipo I (área de silencio) & 60 & 50 \\
Tipo II (área levemente ruidosa) & 65 & 50 \\
Tipo III (área tolerablemente ruidosa) & 70 & 70 \\
Tipo IV (área ruidosa) & 75 & 75 \\
Tipo V (área especialmente ruidosa) & 80 & 70 \\
\hline
\end{tabular}

Fuente: elaborada según los dados de Legislatura de la Ciudad Autónoma de Buenos Aires (Ley 1540, 2004). 
Tabla 3. Límites máximos permisibles de ruido en Río de Janeiro

\begin{tabular}{lcc}
\hline \multicolumn{1}{c}{ Tipos de uso } & \multicolumn{2}{c}{ Valores límite en dB } \\
\cline { 2 - 3 } & Período diurno & Período nocturno \\
\hline Zonas de preservación y conservación en unidades de & 45 & 40 \\
conservación ambiental y zonas agrícolas & 55 & 50 \\
Residencial urbano & 65 & 60 \\
Zonas de negocios, comercio y administración & 70 & 65 \\
Área predominantemente industrial & & \\
\hline
\end{tabular}

Fuente: Municipio de Río de Janeiro (Ley 3268, 2001).

Ley n. 5354 de 1998 expedida por el municipio de Salvador de Bahía. El artículo 3 establece que los niveles máximos de ruido proveniente de cualquier fuente emisora, en áreas residenciales, comerciales, de servicios, institucionales, industriales y especiales, sean públicas o privadas, serán de $60 \mathrm{~dB}$ en el período comprendido entre las 22:00 y 7:00 horas, y de $70 \mathrm{~dB}$, entre las 7:00 y las 22:00 horas (Ley 5354, 1998).

Réglamentation bruit. Ministère de L'Environnement, de l’Énergie et de la Mer [Reglamentación del ruido. Ministerio del Ambiente, la Energía y el Mar]. Los valores permitidos en Francia son $51 \mathrm{~dB}$ para domingos y festivos durante el período diurno (7:00 h-22:00 h), y $49 \mathrm{~dB}$ durante el período nocturno (22:00 h-7:00 h). En los límites entre propiedades, la norma establece un máximo de $70 \mathrm{~dB}$ en el día y $60 \mathrm{~dB}$ en la noche (Ministère de L'Environnement, de l'Énergie et de la Mer, 2006).

Tabla 4. Lineamientos de ruido nocturno para la Unión Europea

\begin{tabular}{ll}
\hline \multicolumn{2}{c}{ Estado miembro de la UE } \\
\hline Alemania & $49 \mathrm{~dB}$ \\
Austria & $50 \mathrm{~dB}$ \\
España & $45 \mathrm{~dB}$ \\
Estonia & $45 \mathrm{~dB}$ \\
Finlandia & $46 \mathrm{~dB}$ \\
Francia & $62 \mathrm{~dB}$ \\
Holanda & $40 \mathrm{~dB}$ \\
Hungría & $55 \mathrm{~dB}$ \\
Letonia & $40 \mathrm{~dB}$ \\
Suecia & $51 \mathrm{~dB}$ \\
Suiza & $50 \mathrm{~dB}$ \\
\hline
\end{tabular}

Fuente: elaboración propia a partir de World Health Organization Europe (2009).

\section{Análisis comparativo}

Es importante subrayar que la normativa colombiana presenta inconsistencias a nivel nacional y regional. A nivel nacional, la Resolución 8321 de 1983 del Ministerio de Salud establece un máximo de $65 \mathrm{~dB}$ diurnos y $45 \mathrm{~dB}$ nocturnos para las zonas residenciales; mientras que la Resolución 627 de 2006 del Ministerio de Ambiente establece un máximo de $65 \mathrm{~dB}$ diurnos y 
$55 \mathrm{~dB}$ nocturnos para estas mismas zonas. Como se observa, las resoluciones no coinciden en el período nocturno.

A nivel regional también hay diferencias notables. La Resolución 6918 de 2010 de la Secretaria de Ambiente de Bogotá establece un máximo de $55 \mathrm{~dB}$ diurnos y $45 \mathrm{~dB}$ nocturnos para las zonas residenciales, mientras que en Medellín se permiten $65 \mathrm{~dB}$ diurnos y $55 \mathrm{~dB}$ nocturnos para estas mismas zonas.

A nivel internacional la diversidad de umbrales permitidos de ruido en zonas residenciales es aún más dispar. Revisando solamente los decibeles permitidos en la fracción diurna para zonas residenciales de seis ciudades, se encuentran grandes diferencias (ver tabla 5).

Tabla 5. Umbrales de ruido permitidos en seis ciudades

\begin{tabular}{ll}
\hline Buenos Aires & $65 \mathrm{~dB}$ \\
París & $70 \mathrm{~dB}$ \\
Perú & $60 \mathrm{~dB}$ \\
Quito & $50 \mathrm{~dB}$ \\
Río de Janeiro & $55 \mathrm{~dB}$ \\
Salvador de Bahía & $70 \mathrm{~dB}$ \\
\hline
\end{tabular}

Fuente; elaboración propia a partir de normativas citadas anteriormente.

En este breve muestreo se evidencia que no existe un consenso en relación al nivel de ruido aceptado en las ciudades, lo que aporta fundamentos para realizar una pesquisa a fondo para establecer parámetros sólidos y coherentes que revisen, renueven y actualicen las normativas nacionales e internacionales en el tema del ruido, $y$, de ser pertinente, presentar una solicitud de cambio en los umbrales de ruido permitidos en torno a los autódromos ante la autoridad ambiental colombiana. De igual manera, resulta urgente y necesario modificar los POT en lo concerniente al establecimiento de zonas de afectación, porque, cuando se otorgan permisos para la construcción de autódromos, la norma actual permite el ruido en el interior de los linderos del escenario deportivo, pero no en su perímetro inmediato, lo que en la práctica es completamente imposible.

\section{Conclusiones}

Desde sus orígenes, los deportes a motor han generado una serie de dinámicas sociales, económicas, culturales y tecnológicas que contribuyen de manera significativa con la sustentabilidad económica de los territorios en donde se desarrollan. En la actualidad, esas dinámicas se concentran en los autódromos, escenarios deportivos que, además de ser los únicos apropiados para los deportes a motor (puesto que permiten una mayor cobertura de los riesgos y un mayor control de la accidentalidad para el público y los pilotos), son polos de atracción turística e importantes focos de actividad económica e innovación tecnológica. Sin embargo y pese a los enormes beneficios que generan a nivel de la salud, la seguridad, la economía, el tejido social y los desarrollos del sector automotor, en muchas ocasiones se ven limitados en su actividad por el ruido que generan las competencias. En consecuencia, resulta prioritario desarrollar artefactos y dispositivos que reduzcan la dispersión del ruido producido por los automotores en el ambiente. De manera paralela a estos desarrollos, es necesario revisar la legislación actual en el tema del ruido; normativa que además de ser inadecuada y obsoleta, obliga a los autódromos a permanecer por debajo de ciertos umbrales de ruido que, de por sí, son de imposible cumplimiento, o a cerrar estos escenarios deportivos en detrimento del desarrollo económico social y tecnológico de muchas ciudades y municipios, y 
de algo aún más importante: en perjuicio de la seguridad en las vías y las calles y poniendo en alto riesgo la vida humana.

\section{REFERENCIAS}

Área Metropolitana del Valle de Aburrá. (2010). Gestión para la prevención y mitigación del ruido urbano. Recuperado de www.metropol.gov.co/CalidadAire//sdocRuido/gua_ambiental_comunidad.pdf

Añó, V., Bastías, D., y Pablos, C. (2010). Percepción social del Gran Premio de Europa de Fórmula 1 entre los ciudadanos de Valencia. European Journal of Human Movement, 25, 143-164.

Bies, D., y Hansen, C. (2009). Engineering noise control. Theory and practice. Nueva York: Spon Press.

Cai, R., Thong, M., Ong, W., Chan, M., Ng, Y., y Phua, G. (2013). Industrial noise reduction. Singapur: School of Mechanical \& Aeronautical Engineering.

Carrado, A. (2015). Evaluación de la contaminación sonora en el kartódromo de Ciudad Evita, Partido de La Matanza (tesis). Universidad del Salvador, Buenos Aires, Argentina.

Cheng, E., y Jarvis, N. (2010). Residents' perception of the social-cultural impacts of the 2008 Formula 1 Singtel Singapore Grand Prix. Event Management, 14(2), 91-106.

Cobo, P., Bravo, M., Cuesta, M., Ranz, C., y Siguero, M. (2000). Control activo del ruido. Revista de Acústica, 31(3-4), 1-4.

Concejo Metropolitano de Quito. (5 de julio de 2004). Ordenanza para prevención y control de la contaminación sustitutiva del capitulo II para el control del ruido, del titulo V del libro segundo del Código Municipal. [Ordenanza Metropolitana n. 0123 de 2004]. Recuperado de http://www7. quito.gob.ec/mdmq_ordenanzas/Ordenanzas/ORDENANZAS\%20A\%C3\%91OS\%20 ANTERIORES/ORDM-123\%20-\%20RUIDO\%20-\%20MEDIO\%20AMBIENTE.pdf.

Consejo Municipal de Rosario. (11 de julio de 2012). [Ordenanza n. ${ }^{\circ} 8934$ de 2012]. Recuperado de https://www.rosario.gov.ar/normativa/verArchivo;jsessionid=E23C2ED092459230ED B4113FD2CE874E.nodo14_tomcat04? tipo=pdf\&id $=84880$

Corte Constitucional. (1995). Sentencia T-226 de 1995. [MP Fabio Moron Díaz]. Recuperado de http://www.corteconstitucional.gov.co/relatoria/1995/T-226-95.htm.

Côté, R. (2013). Autodrome Saint-Eustache: Mise sur pied d'un comité de vigilance Autodrome-citoyens. L'Éveil et La Concorde. Recuperado de http://www.leveil.com/actualites/ autodrome-saint $\% \mathrm{E} 2 \% 80 \% 91$ eustache-mise-sur-pied-dun-comite-de-vigilance-autodrome-citoyens- 2.

Dingle, G. (2009). Sustaining the race: a review of literature pertaining to the environmental sustainability of motorsport. International Journal of Sports Marketing and Sponsorship, 11(1), 75-91.

Elliott, S., y Nelson, P. (1993). Active noise control. Signal Processing Magazine, 10(4), 12-35.

Fang, Ch-F., y Ling, D-L. (2003). Investigation of the noise reduction provided by tree belts. Landscape and Urban Planning, 63(4): 187-195.

Gana, F. (2014). Corte Suprema rechaza recurso de protección por Autódromo Internacional de Codegua. Racing5. Recuperado de http://www.racing5.cl. 
German-González, M., y Santillán, A. (2006). Del concepto de ruido urbano al de paisaje sonoro. Bitácora Urbano Territorial, 10(1), 39-52.

Henderson, J., Foo, K., Lim, H., y Yip, S. (2010). Sports events and tourism: The Singapore formula one grand prix. International Journal of Event and Festival Management, 1(1), 60-73.

Hunashal, R., y Patil, Y. (2012). Assessment of noise pollution indices in the city of Kolhapur, India. Procedia-Social and Behavioral Sciences, 37, 448-457.

Kardous, C., y Morata, T. (2010). Occupational and recreational noise exposures at stock car racing circuits: An exploratory survey of three professional race tracks. Noise Control Engineering Journal, 58(1), 54-61.

Kuo, S., y Morgan, D. (1999). Active noise control: a tutorial review. Proceedings of the IEEE, 87(6), 943-973.

Legislatura de la Ciudad Autónoma de Buenos Aires. (2 de diciembre de 2004). Control de la contaminación acústica en la Ciudad Autónoma de Buenos Aires. [Ley n. ${ }^{\circ} 1540$ de 2004]. Recuperado de: http://www.pensamientopenal.com.ar/system/files/2014/12/legislacion32902.pdf.

Lueg, P. (1936). Patente US2043416. Recuperado de www.google.com/patents/US2043416.

Maleki, K., y Hosseini, S. (2011). Investigation of the effect of leaves, branches and canopies of trees on noise pollution reduction. Annals of Environmental Science, 5, 13-21.

Ministère de L'Environnement, de l'Énergie et de la Mer. (2006). Réglementation bruit. Recuperado de http://www.installationsclassees.developpement-durable.gouv.fr/3-Reglementation-bruit.html.

Ministerio de Ambiente, Vivienda y Desarrollo Territorial. (7 de abril de 2006) Por la cual se establece la norma nacional de emisión de ruido y ruido ambiental. [Resolución 627 de 2006]. DO: 46.239 .

Ministerio del Medio Ambiente. (5 de junio de 1995) Prevención y control de la contaminación atmosférica y la protección de la calidad del aire. [Decreto 948 de 1995]. DO: 41.876.

Ministerio de Salud. (4 de julio de 1983). Protección y conservación de la audición de la salud y el bienestar de las personas, por causa de la producción y emisión de ruidos. [Resolución 8321 de 1983]. Recuperado de: http://www.alcaldiabogota.gov.co/sisjur/normas/Norma1.jsp?i=6305.

Miyara, F. (1999). Control del ruido. Recuperado de: http://www.ingenieroambiental.com/4023/ control\%20de\%20ruido,federico\%20miyara.pdf.

Municipio do Río de Janeiro. (29 de agosto de 2001). Proteção da coletividade contra a poluição sonora. [Ley n. 3268 de 2001]. Recuperado de http://mail.camara.rj.gov.br/APL/Legislativos/ contlei.nsf/bff0b82192929c2303256bc30052cb1c/72ffac703b58de4a032576ac0072e89c?O penDocument\#_Section2.

Município de Salvador de Bahia. (28 de enero de 1998). Dispóe sobre sons urbanos, fixa niveis e horários em que será permitida sua emissão, cria a licença para utilização sonora e dá outras providências. [Ley n. 5354 de 1998]. Recuperado de http://www.sucom.ba.gov.br/wp-content/ uploads/2014/11/lei5354_1998.pdf.

Paredes, M. (2016). Autódromo de Codegua es nuevamente clausurado. AS. Recuperado de http:// chile.as.com/chile/2016/01/19/masdeporte/1453158652_342172.html. 
Pfretzschner, J., Simón, F., Rodriguez, R., y Moreno, A. (2010). Barreras acústicas. Revista de Acústica. Instituto de Acuistica, 32(1-2), 2-5.

Presidencia de la República del Perú. (30 de octubre de 2003). Reglamento de estándares nacionales de calidad ambiental para ruido. [Decreto Supremo n. ${ }^{\circ} 085$ de 2003-PMC]. Recuperado de http://www.minam.gob.pe/calidadambiental/wp-content/uploads/sites/22/2013/10/ DS.085.2003.PCM_.pdf.

Secretaría Distrital de Ambiente. (19 de octubre de 2010). Por la cual se establece la metodología de medición y se fjan los niveles de ruido al interior de las edificaciones (inmisión) generados por la incidencia de fuentes fijas de ruido. [Resolución 6918 de 2010]. Recuperado de: http://www. alcaldiabogota.gov.co/sisjur/normas/Norma1.jsp?i=40790.

Seneca, M. (2003). The Fairmount Park Motor Races 1908-1911. Jefferson/London: McFarland y Company.

Serrano, P., Abad, T., Magro, A., y García, M. (2009). Estudio de la tipología de las pantallas acústicas. Normativa y estado del arte. Revista Tecnología y Desarrollo, 7, 2-43.

Thomas, P., Dragonetti, L., van Renterghem, T., y Botteldooren, D. (2010). Detailed analysis of the sound field in a scale model of a street canyon. Conferencia presentada en $1^{\text {st }}$ Congress on Sound and Vibration, Ljubljana, Slovenia.

Tranter, P. (2003). Motor racing in Australia: health damaging or health promoting? Australian Journal of Primary Health, 9(1), 50-58.

Tsai, K., Lin, M., y Chen, Y. (2009). Noise mapping in urban environments: A Taiwan study. $A p^{-}$ plied Acoustics, 70(7), 964-972.

Van Renterghem, T., Botteldooren, D., y Verheyen, K. (2012). Road traffic noise shielding by vegetation belts of limited depth. Journal of Sound and Vibration, 331(10), 2404-2425.

Van Renterghem, T., Forssén, J., Attenborough, K., Jean, P., Defrance, J., Hornikx, M., y Kang, J. (2015). Using natural means to reduce surface transport noise during propagation outdoors. Applied Acoustics, 92, 86-101.

Vogiatzis, K. (2012). Airport environmental noise mapping and land use management as an environmental protection action policy tool. The case of the Larnaka International Airport (Cyprus). Science of the Total Environment, 424, 162-173.

World Health Organization Europe (2009). Night noise guidelines for Europe. Copenhague: World Health Organization.

Yang, W., y Kang, J. (2005). Acoustic comfort evaluation in urban open public spaces. Applied Acoustics, 66(2), 211-229.

Yepes, D., Gómez, M., Sánchez, L., y Jaramillo, A. (2009). Metodología de elaboración de mapas acústicos como herramienta de gestión del ruido urbano-caso. Dyna, 158, $29-40$.

Zannin, P., y de Sant'Ana, D. (2011). Noise mapping at different stages of a freeway redevelopment project-A case study in Brazil. Applied Acoustics, 72(8), 479-486. 
